\title{
Can muscle protein metabolism be specifically targeted by exercise training in COPD?
}

\author{
Davina C. M. Simoes ${ }^{1}$, Ioannis Vogiatzis ${ }^{2}$ \\ ${ }^{1}$ Department of Applied Sciences, ${ }^{2}$ Department of Sport, Exercise and Rehabilitation, Faculty of Health and Life Sciences, Northumbria \\ University, Newcastle-upon-Tyne, UK \\ Contributions: (I) Conception and design: All authors; (II) Administrative support: None; (III) Provision of study materials or patients: None; (IV) \\ Collection and assembly of data: None; (V) Data analysis and interpretation: None; (VI) Manuscript writing: All authors; (VII) Final approval of \\ manuscript: All authors. \\ Correspondence to: Davina C. M. Simoes. Department of Applied Sciences, Faculty of Health and Life Sciences, Northumbria University, Newcastle- \\ upon-Tyne, UK. Email: davina.simoes@northumbria.ac.uk.
}

\begin{abstract}
Patients with stable chronic obstructive pulmonary disease (COPD) frequently exhibit unintentional accentuated peripheral muscle loss and dysfunction. Skeletal muscle mass in these patients is a strong independent predictor of morbidity and mortality. Factors including protein anabolism/catabolism imbalance, hypoxia, physical inactivity, inflammation, and oxidative stress are involved in the initiation and progression of muscle wasting in these patients. Exercise training remains the most powerful intervention for reversing, in part, muscle wasting in COPD. Independently of the status of systemic or local muscle inflammation, rehabilitative exercise training induces up-regulation of key factors governing skeletal muscle hypertrophy and regeneration. However, COPD patients presenting similar degrees of lung dysfunction do not respond alike to a given rehabilitative exercise stimulus. In addition, a proportion of patients experience limited clinical outcomes, even when exercise training has been adequately performed. Consistently, several reports provide evidence that the muscles of COPD patients present training-induced myogenic activity limitation as exercise training induces a limited number of differentially expressed genes, which are mostly associated with protein degradation. This review summarises the nature of muscle adaptations induced by exercise training, promoted both by changes in the expression of contractile proteins and their function typically controlled by intracellular signalling and transcriptional responses. Rehabilitative exercise training in COPD patients stimulates skeletal muscle mechanosensitive signalling pathways for protein accretion and its regulation during muscle contraction. Exercise training also induces synthesis of myogenic proteins by which COPD skeletal muscle promotes hypertrophy leading to fusion of myogenic cells to the myofiber. Understanding of the biological mechanisms that regulate exercise training-induced muscle growth and regeneration is necessary for implementing therapeutic strategies specifically targeting myogenesis and hypertrophy in these patients.
\end{abstract}

Keywords: Chronic obstructive pulmonary disease (COPD); exercise; protein synthesis; anabolism; hypertrophy; myogenesis; muscle wasting

Submitted Nov 27, 2017. Accepted for publication Feb 22, 2018.

doi: $10.21037 /$ jtd.2018.02.67

View this article at: http://dx.doi.org/10.21037/jtd.2018.02.67

\section{Introduction}

Chronic obstructive pulmonary disease (COPD) is the fourth leading cause of death worldwide and is projected to be the third most common cause of death by 2020 (1).
Cigarette smoke constitutes the major preventable risk factor, resulting in a progressive proteolytic, inflammatory and vasoactive response that leads to emphysema, small airway obstruction and pulmonary hypertension. The 
oxidative stress imposed by cigarette smoking together with systemic inflammation and hypoxia are important contributors to pathogenesis of skeletal muscle wasting and dysfunction and have been previously extensively reviewed (2-4). Skeletal muscle wasting is an important systemic effect of the disease and a strong independent predictor of mortality (5-7). This unintentional accentuated skeletal muscle wasting is frequently associated with altered muscle structure (fiber size, fiber type distribution, capillary density and metabolic capacity) and dysfunction (decreased strength and endurance). While the magnitude of the alterations varies substantially across individuals, some degree of muscle wasting affects all individuals during ageing. Age-related defects in protein metabolism have been proposed to be causally involved in this muscle loss (8). These changes are attributed to both inactivity- and agerelated alterations in protein synthesis and degradation, indicating complex pathophysiological phenomena involving both structural changes to the muscle fibres, as well as the enzymatic machinery that controls metabolism. Independently of the factors promoting muscle wasting in COPD, regular physical exercise remains the most potent available treatment option for reversing, in part, locomotor muscle wasting and dysfunction in COPD (9). Indisputably, exercise training promotes a range of beneficial adaptations in the skeletal muscle including increased capillarization, fibre type plasticity, hypertrophy and function (9). However, exercise training induced muscle benefits are certainly much smaller in COPD patients compared to age matched controls. In addition, not all COPD patients respond adequately to exercise stimulus, even when exercise training is properly performed $(10,11)$. An improved understanding of molecular mechanisms of exercise induced adaptations in COPD and healthy individuals will be valuable to inform future directions to address the issues on limitation to exercise-induced adaptations in COPD.

\section{Effect of prescribed exercise training to skeletal muscle adaptation in COPD}

Exercise training is a mechanical stimulus that consists of repeated, episodic bouts of muscle contraction promoting functional adaptation and remodelling not only to the skeletal muscle, but also to various systems in our body $(12,13)$. Exercise promotes a range of adaptations that is beyond the musculoskeletal system promoting general health (14). Briefly, in parallel with neural signals to the skeletal muscle contraction, powerful neural feed-forward signals to the respiratory, cardiovascular, metabolic and hormonal systems are produced. In response to exercise training, COPD patients demonstrate reduction in dyspnea sensations, improvements in exercise capacity and quality of life (9).

Skeletal muscle adaptations are dependent on the intensity and duration of the exercise training performed (15). High intensity exercise training (Wpeak $\geq 80 \%$ ) is generally described to promote improvement in exercise capacity. However, COPD patients with limited ventilatory capacity are usually unable to sustain high intensities for sufficiently long periods. Taking into consideration COPD patients exercise capacity, a number of studies have been performed employing a varied combination of exercise modalities/training, program duration and intensity. Usually, the duration of an exercise training programme is set from 8 to 12 weeks and as frequent as 3 times per week. Combination of high intensity aerobic and resistance exercise is described to promote quantifiable muscle hypertrophy in COPD $(10,11,16,17)$. Conversely, exercise training of lower intensity (Wpeak $\leq 60 \%$ ) was found unable to promote quantifiable changes in muscle hypertrophy and fibre type distribution $(18,19)$. Therefore, programmes incorporating high intensity exercise training are more likely to induce quantifiable skeletal muscle adaptations.

Different modes of exercise such as endurance- and resistance-based are known to stimulate variable but specific skeletal muscle adaptations, leading to muscle endurance and strength respectively (15). Aerobic/endurance exercise training enhances mitochondrial protein content and oxidative capacity of trained myofibers, improving insulin sensitivity and skeletal muscle metabolic function (20). Whereas, resistance training increases myosin-heavychain gene transcripts and synthesis rate of muscle proteins promoting strength (15). When comparing different modalities of exercise prescribed to healthy sedentary people, Robinson et al. (21) observed that high intensity interval training (HIIT) enhanced more comprehensively changes such as aerobic capacity, mitochondria respiration and lean body mass (21). HIIT training simultaneously promoted endurance- and resistance-based training skeletal muscle adaptations, that promoted changes in transcription and translation regulation of muscle growth and mitochondrial pathways (21). HIIT reversed age-related proteome, particularly of mitochondrial proteins. But both resistance training and HIIT increase proteins involved in translational machinery. HIIT exercise involves 30-120 s 


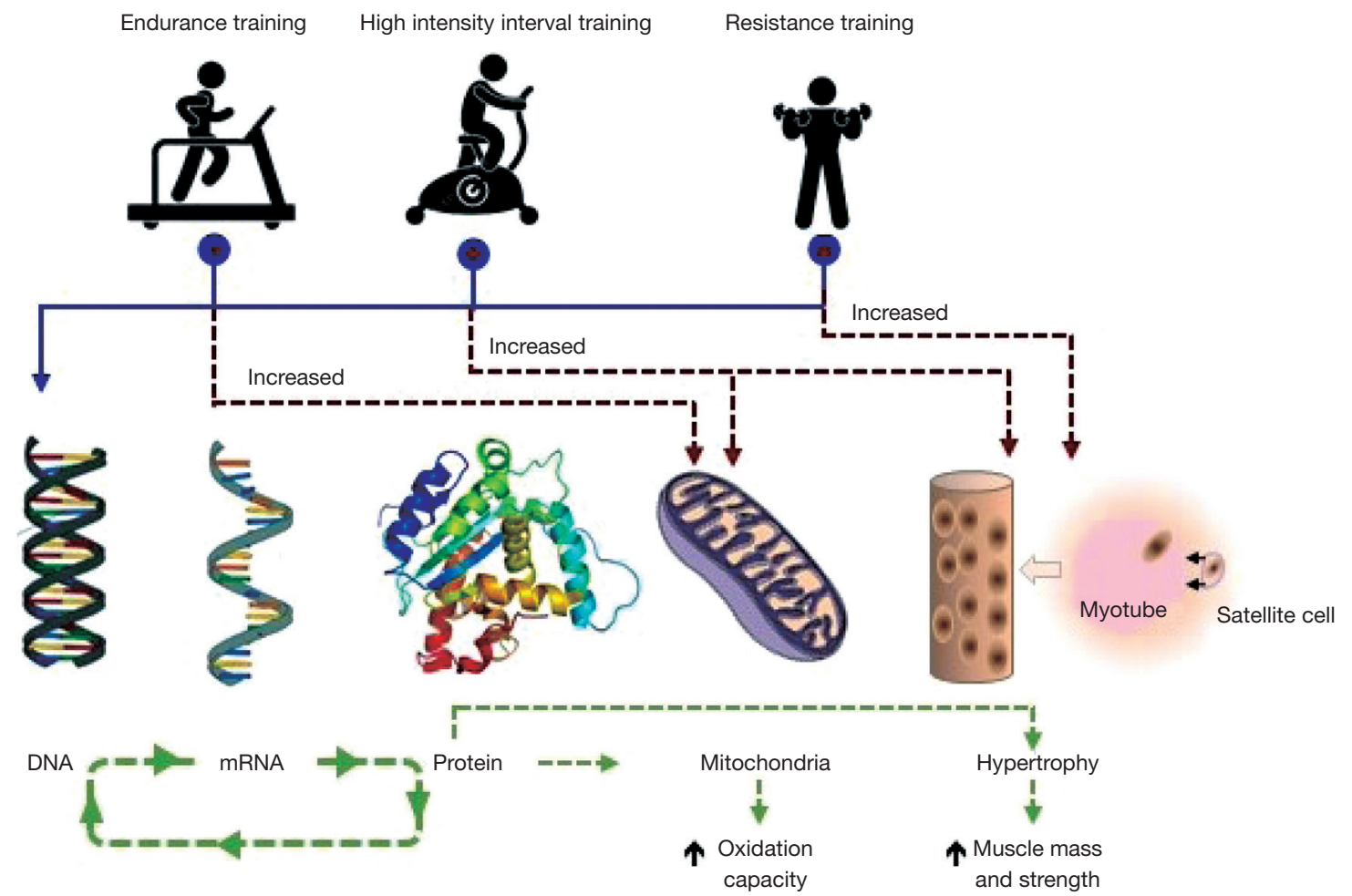

Figure 1 COPD skeletal muscle adaptations to various modalities of exercise training. Exercise training is a powerful stimulus producing hypertrophy and regeneration of muscle by increased protein metabolism and fusion of satellite cells to existent myofiber. Enduranceand resistance-based exercise training programmes are characterised as stimuli capable for increasing oxidative capacity and hypertrophy, respectively, whereas high intensity interval training is capable of increasing both oxidative capacity and hypertrophy. Skeletal muscle adaptations observed from combined endurance/resistance as well as high intensity interval training reflect a wider range of stimuli. [The Figure has been inspired by Robinson et al. (21) and modified based on presented theory]. COPD, chronic obstructive pulmonary disease.

repeated short bouts of activity at near maximal intensity (Wpeak $280 \%$ ) interspersed with $30-120$ s rest periods, which despite its high intensity, has been successfully applied to COPD patients $(10,11,17,22)$. A representation of basic skeletal muscle adaptation promoted by aerobic/ endurance, resistance and HIIT is depicted in Figure 1.

Therefore, adaptations seen in COPD skeletal muscle take place in accordance with specific exercise training stimuli, thereby partially explaining the large variability in muscle adaptations seen among studies. The effect of exercise modalities in promoting COPD skeletal muscle adaptations at structural and protein metabolism levels have been recently presented in a systemic review (19).

Histologically, the skeletal muscle appears uniform, but is composed by a range of heterogeneous myofibers regarding size, metabolism and contractile function. When comparing myosin-heavy-chain isoform expression, myofibers are classified into types I, IIa, IId/x, and IIb fibres (23). Types I and IIa fibers exhibit high oxidative potential and IIx and IIb are primarily glycolytic. The degree to which enduranceor resistance-based exercise training can induce myofiber plasticity is still debatable. Certainly, COPD patients present a shift in fibre type displaying fewer type I (oxidative) fibres and greater proportion of type II (glycolytic) fibres in quadriceps muscles (24). This shifting towards glycolytic fibres is associated with increased mortality (7). Exercise training prescribed to COPD patient can only partially reverse this fiber type shifting. Proportion of fiber types I and IIa were increased mainly after HIIT and high intensive aerobic exercise $(19,25)$ as endurance training confers an increased oxidative profile to trained myofibers $(9,11,22,23)$. Hypertrophy of fibre types I and IIA was more widespread among different modalities of exercise training $(9,19)$, whereas, capillary to fiber ratio adaptations were though observed across various intensities and modalities of exercise training $(9,19)$. 


\section{Contribution of inactivity to COPD muscle wasting}

Inactivity appears to be an important mechanism in the process of muscle wasting in COPD, given that muscles that are active, such as the diaphragm and the adductor pollicis, do not exhibit atrophy in contrast to inactive muscles, such as the quadriceps (26). In experiments comparing different muscle groups in COPD patients, the characteristics exhibited by the deltoid and the diaphragm were different compared to the quadriceps (27). Importantly, the muscles of respiration in COPD exhibit a contrary shift in fiber typing compared to the locomotor muscles, manifested by increased type I fiber distribution $(28,29)$. Various conditions of reduction in neuromuscular activity promoted by inactivity are known to decrease myonuclear number in atrophying muscle and impact on fibre typing. As reviewed elsewhere (30), detraining experiments in healthy individuals have shown to induce locomotor muscle adaptations that lead to increased number of muscle fibre type IIx phenotype, and attenuation of mitochondrial biogenesis [peroxisome proliferator-activated receptors (PPARs) and PGC-1 $\alpha$ ] as observed in COPD patients. As described above, resuming activity by exercise training can partially promote changes in fibre type distribution (19). However, inactivity alone does not seem to fully explain the phenomenon of muscle fibre type shifting in COPD.

\section{Mechanical stress and mitogen-activated protein kinase (MAPK) signalling}

The ability of skeletal muscles to respond to physical exercise by executing the appropriate metabolic and transcriptional response is dependent upon the cellular signal transduction through phosphorylation cascades. Multiple kinases, including AMPK, Akt and the MAPKs are involved in the regulation of DNA transcription through the phosphorylation of nuclear transcription factors. This either enhances or inhibits the ability of transcription factors to bind DNA, affecting target gene transcription $(31,32)$. Three main MAPK subfamilies are activated by acute exercise in human skeletal muscle: (I) the extracellularregulated kinase (ERK1/2), (II) the c-jun N-terminal kinase (JNK), and (III) the p38 MAPK. Activation of MAPKs regulates the transcriptional events by phosphorylation of diverse substrates localised in the cytoplasm or nucleus, including transcription factors, inducing differentiation, hypertrophy, inflammation, and gene expression (33).
The MAPK p38 is a stress-activated kinase that is transiently activated in response to a strenuous range of stimuli such as physical inactivity and increased intensity of exercise training $(34,35)$. Activation of $\mathrm{p} 38$ in skeletal muscle myoblasts is related to loss in satellite cell autonomous selfrenewal capacity (36). MAPK p38 activation is also observed during skeletal muscle immobilisation in a rat hind limb model of acute muscle wasting $(34,37)$. COPD are generally more inactive compared to their age-matched healthy counterparts (38). Accordingly, ratios of phosphorylated to total level of p38 MAPK and ERK 1/2 were significantly elevated in patients with COPD compared to controls (39). Whereas, another study has shown no differences in the ratio of phospho-p38 MAPK to total level of p38 MAPK protein between COPD patients and healthy age-matched donors. Although patients with COPD present muscle wasting, discrepancies among studies would be expected as it is uncertain whether patients are actively losing muscle mass at the time of experimentation.

\section{Major signaling pathways involved in the control of exercise training induced skeletal muscle adaptations}

Endurance- and resistance-based modalities of exercise are controlled by two major signaling pathways regulating mitochondria biogenesis and hypertrophy, respectively as depicted in Figure 2.

The regulation of mitochondrial biogenesis by endurance-based exercise converge from activation of the cascades AMPK and p38 upregulating PGC- $1 \alpha$. When compared to healthy controls, mitochondria density is lower in quadriceps muscle of COPD patients, presenting lower expression of PPARs, PPAR- $\gamma$ co-activator $1 \alpha$ (PGC- $1 \alpha)$ and mitochondrial transcription factor (TFAM) in cachectic COPD (40). In patients with COPD, exercise enhances the decrease in mitochondria DNA content of skeletal muscle and the expression of PGC- $1 \alpha$ mRNA seen in healthy subjects, probably due to oxidative stress (41).

In contrast, resistance training is described to stimulate the signaling pathways responsible for muscle hypertrophy $(12,13)$. The activation of mTOR and IGF-I appears to be important in this process. To restore muscle mass via regular exercise training, protein synthesis should exceed protein breakdown over an extended period. Hypertrophy of skeletal muscle as result of resistance exercise training is strongly associated with the degree of mTOR activation, ribosomal protein $\mathrm{S} 6 \mathrm{~K}\left(\mathrm{p} 70^{\mathrm{S} 6 \mathrm{~K}}\right)$ phosphorylation and 


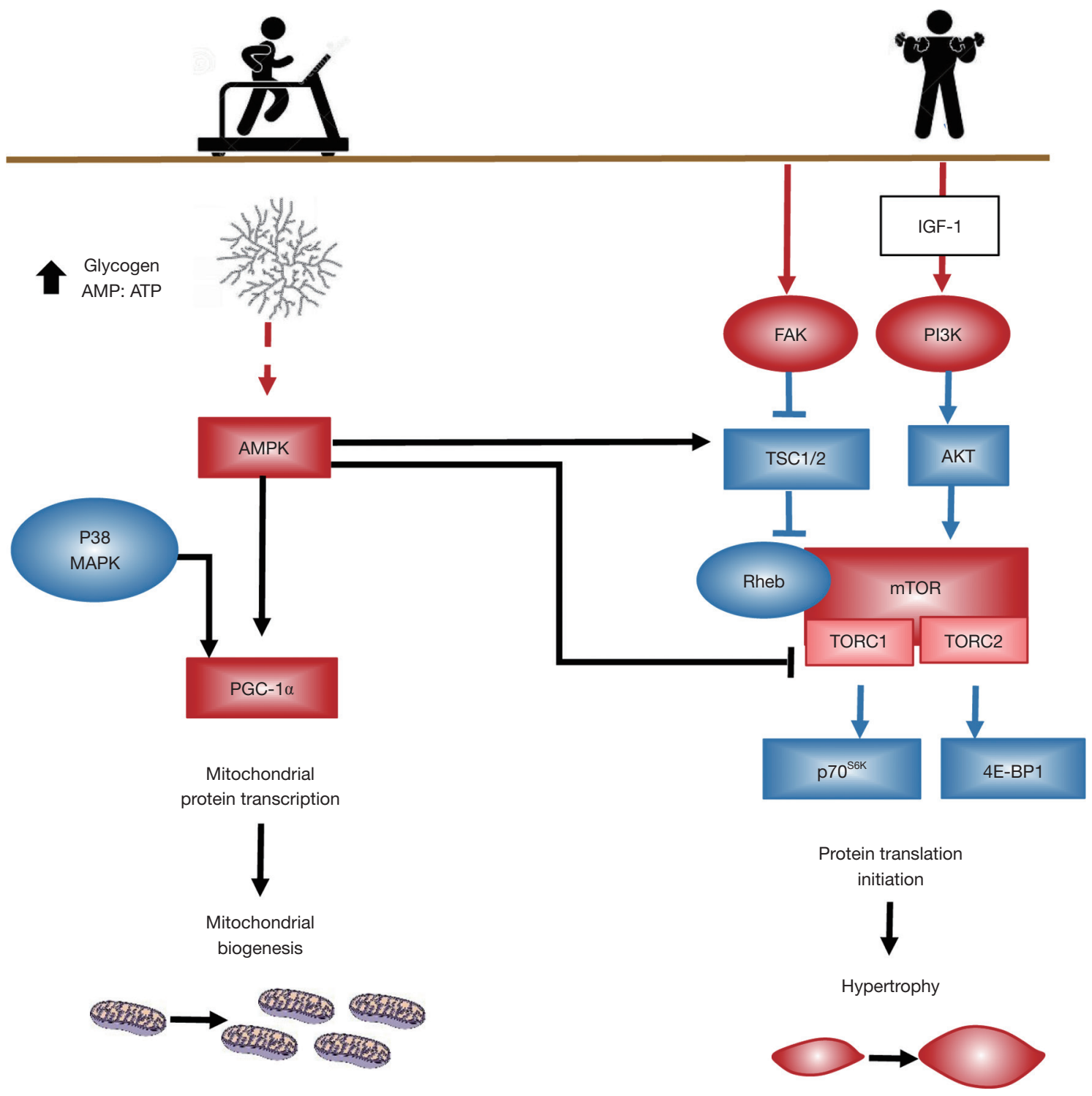

Figure 2 Diagram of the major signalling pathways involved in the control of skeletal muscle hypertrophy and mitochondrial biogenesis. Voluntary exercise training activates kinases/phosphatases to mediate a specific exercise-induced signal. The cross talk among the numerous signalling pathways activated and the multiple site regulation produces a high sensitive and complex transduction network. Activation of AMPK by aerobic/endurance exercise training enhances mitochondrial biogenesis partly by directly phosphorylating and activating PGC$1 \alpha$. Resistance training is a potent stimulus for the increase in skeletal muscle mass. Activation of FAK through integrins leads to the inhibition of TSC, thereby permitting activation of mTOR. In addition, hypertrophy is promoted by activation of IGF-1 activating PI3K/ Akt/mTOR signaling pathway that leads to hypertrophy. AMPK, AMP-activated protein kinase; PGC-1 $\alpha$, PPAR- $\gamma$ coactivator $1 \alpha$; FAK, focal adhesion kinase; TSC, tuberous sclerosis complex; mTOR, mammalian target of rapamycin; IGF-1, insulin-like growth factor 1; PI3K, phosphatidylinositide 3-kinases.

downstream targets (42). Contraction-induced $\mathrm{p} 70^{\mathrm{S} 6 \mathrm{~K}}$ activation is dependent on mTOR activation, which increases protein translation and inhibits protein degradation via inhibition of both ubiquitin-proteasome
(UP) $(32,43,44)$ and autophagy-lysosome (AL) pathways $(45,46)$. mTOR activation is critical to load-induced muscle growth, as demonstrated by the attenuation of hypertrophy responses and protein synthesis by the mTOR inhibitor, 
rapamycin (43).

We have described that Akt/mTOR pathway is downregulated in skeletal muscle of non-cachectic COPD patients compared to cachectic (10). HIIT promotes the activation of the Akt/mTOR pathway in skeletal muscle only in COPD patients with preserved muscle mass compared to cachectic (10). Some studies suggest that hypoxemia characteristically observed in more severe cachectic COPD patients, is associated with resistance of skeletal muscle activation of the Akt/mTOR pathway (16). Exercise training in hypoxemic patients with COPD was not capable to promote muscle fibre hypertrophy and activation of the Akt/mTOR pathway as compared to normoxemic COPD patients (16). Both in vitro C2C12 myotubes cultured in normoxic and hypoxic conditions and mice models of hypoxia suggest that the response of the $\mathrm{Akt} / \mathrm{mTOR}$ pathway to exercise could be compromised in hypoxemic patients $(16,47)$. Therefore, impairment of skeletal muscle hypertrophy commonly linked to the severity of the disease is associated with the magnitude of muscle wasting, the degree of hypoxia, or both. Interestingly, induced expression of the adaptive response of hypoxia HIF-1 responsive RTP801 (DDIT4) is observed only in trained COPD and not in healthy subjects (48).

mTOR regulates the mechanisms of protein synthesis at several levels (e.g., translation capacity, translation efficiency) through increases of translation of specific mRNAs, which culminates in skeletal muscle fibre enlargement. mTOR exists as part of two multi-protein complexes: (I) mTORC1, which contains raptor and confers rapamycin sensitivity, is required for signalling to $\mathrm{p} 70^{\mathrm{S} 6 \mathrm{~K}}$ and 4E-BP1; whereas (II) mTORC2, which contains Rictor and is rapamycin insensitive, is required for signalling to AktFOXO (49). The effect of mTOR activity on downstream regulators of protein synthesis is principally achieved through a contraction-induced regulation of mTORC1 (50).

Early work on adaptive hypertrophy has focused on the (transient) post exercise rise in blood-borne anabolic hormones, such as growth hormone and insulin-like growth factor-I (IGF-I), and the consequent activation of the muscle protein synthesis of a signalling cascade [phosphatidylinositol 3-kinase (PI3K)-Akt-mTOR] by IGF-I interaction with insulin and IGF receptors (51). Recently, the muscle growth paradigm has shifted focus to IGF-I-independent mechanisms of mTOR activation and adaptive hypertrophy through mechano-sensory regulation (50). Nutrient-dependent regulation of muscle growth is achieved through insulin- and Akt-dependent activation of the mTOR pathways. These pathways operate synergistically causing muscle growth and can be augmented by appropriate nutritional intake such as post exercise carbohydrate and amino acid ingestion or increased dietary protein (52). Therefore, IGF-I is involved not only in hypertrophy but also in promoting myogenesis and muscle regeneration.

\section{Regulation of skeletal muscle protein synthesis promoting myogenesis and muscle regeneration}

Skeletal muscle hypertrophy is achieved by both positive protein balance and fusion of satellite cells to myofibers (Figure 1). It is a process that involves (I) accretion of protein in various cellular compartments via mechanosensitive signalling pathways that drive translation; and (II) the activation and recruitment of resident muscle stem cell (satellite cells) that differentiate to fusioncompetent myoblasts $(53,54)$. Regulation of protein translation and synthesis promotes accretion, whereas activation and incorporation of satellite cells facilitates the addition of myofibrils to the muscle.

Myogenesis and muscle regeneration depend on critical steps for activation of quiescent satellite cell, proliferation, migration, differentiation, fusion and maturation. Fusion of a satellite cell to an existent myofiber results in an increase in the number of myonuclei, and thus the available total amount of genetic machinery for protein production (54). The average number of myonuclei per muscle fibre of noncachectic COPD is twice as high compared to controls, indicating higher capacity of protein metabolism necessary for maintenance of muscle mass (55). Quiescent satellite cells are essential to replenishment of myonuclei pool. As satellite cells replicate throughout lifespan, telomeres are shortened. Telomere shortening is a marker of senescence. COPD patients, despite the observation that satellite cells numbers are unaltered in the limb muscle compared to controls, satellite cells present shorter telomeres. A fact suggesting exhausted muscle regenerative capacity, compromising the maintenance of muscle mass (55).

Satellite cells myogenic regulatory factors (MRF) and myostatin play important roles in myogenesis and muscle regeneration. mRNA and protein expression of the myogenic differentiation factor D (MyoD), involved in the proliferation process, has been shown to increase in the skeletal muscle of non-cachectic COPD patients after HIIT, but no changes were observed in cachectic COPD patients after HIIT (10). MyoD protein expression was also 
increased after resistance training with or without nutritional supplementation in patients and healthy controls (56). mRNA expression of MRF myogenin, involved in the differentiation process, was not different between a resistance training group and a control group, while addition of testosterone supplementation to resistance training increased myogenin mRNA expression compared to the control group (57). mRNA and protein expression of the myogenic inhibiting factor myostatin, showed no significant change after resistance exercise training neither in patients nor in healthy controls, or after combined aerobic and resistance training (16,56). After HIIT however, non-cachectic COPD patients showed a significant decrease in mRNA and protein expression of myostatin, while in cachectic COPD patients HIIT did not significantly alter mRNA or protein myostatin expression (10). In addition, mRNA expression of a negative regulator of cell proliferation Kruppel-like factor 10 (KLF10) was increased after aerobic training in patients, but not in healthy controls (48).

The IGF-I system plays an important role in the regulation of muscle cell growth, muscle cell proliferation and muscle cell survival $(58,59)$. After HIIT, mRNA expression of both IGF-I and the mechano-growth factor (MGF), an isoform of IGF-I, significantly increases. Both cachectic and non-cachectic COPD patients have shown enhanced MGF mRNA expression post-training (10). However, no significant increase in protein expression of IGF-I and MGF expression was observed in cachectic COPD patients in comparison to non-cachectic (10). Other exercise protocols such as combined aerobic and resistance training were not capable to increase the expression of the IGF-I variants (16). However, testosterone supplementation was found to increase the levels of IGF-I and MGF protein expression in COPD patients after resistance training (57).

\section{Exercise induced changes in gene expression}

Comparing gene transcription among different modalities of exercise in healthy individuals, Robinson et al. (21) found that HIIT promotes a stronger increase in gene transcripts than other modalities of exercise tested, particularly in older adults.

Skeletal muscle gene expression from COPD after high intensity aerobic exercise training compared to agematched controls were analysed using GeneChip Array (48). High aerobic exercise training induced up-regulation of quantitatively significantly fewer genes in the skeletal muscle of COPD (107 were upregulated and 124 were downregulated) compared with healthy controls (258 were upregulated and 315 were downregulated). Qualitatively, genes associated with protein degradation, such as oxidative stress, ubiquitin proteasome, and COX pathways were distinctly induced only in patients with COPD, potentially reflecting the specific molecular response of the muscle to exercise in COPD, thereby suggesting additional operating mechanisms for exercise limitation in these patients (48). Whether exercise training can sufficiently enhance muscle hypertrophy to outstrip muscle wasting in COPD patients with substantial muscle loss, remains an unresolved issue.

\section{Regulation of skeletal muscle protein breakdown}

Muscle tissue homeostasis is maintained by a tight and complex balance between protein synthesis and degradation. Protein metabolism turnover is a dynamic process balancing protein synthesis and breakdown. Muscle wasting due to an increase in protein breakdown is a feature shared among many acute and chronic disease entities as well as healthy ageing.

Muscle wasting has primarily been attributed to increased protein degradation. Protein degradation in COPD patients peripheral muscles takes place through four proteolytic systems, including the UP pathway, the calpain pathway, the caspase pathway, and the AL pathway as reviewed elsewhere (60).

Exercise has been found to stimulate MAPK-9 and MAPK activated protein kinase 3 (MAPKAPK-3) in COPD compared to healthy controls (48). The MAPK pathway, in turn activates forkhead transcription factors, involved in muscle protein degradation. When inflammatory response to exercise is limited, the muscle recovers in a timely manner; however, persistent systemic inflammation described in COPD, may be associated with muscle wasting and adversely impact on muscle protein metabolism (61). Numerous pathological indicators in COPD, namely systemic inflammation, hypoxia and oxidative stress most likely trigger catabolic processes in skeletal muscle, that are mediated by transcriptional regulators including nuclear factor kappa-light-chain-enhancer of activated B cells (NF-KB) and forkhead box $\mathrm{O}$ transcription factors (FOXOs). The activity of NF- $\kappa \mathrm{B}$ is increased in COPD compared with healthy age-matched individuals $(9,10,19,22,62)$ and in particular in patients with muscle wasting compared to those without muscle wasting $(10,63)$. FOXO mRNA and protein expression is increased in patients with COPD 
$(62,64-67)$. The expression of FOXO-1 may be associated with physical inactivity as protein expression is increased in lower limbs compared to respiratory muscles in COPD patients, but not in healthy controls (68). Increased catabolic signaling through FOXO and NF-kB activation may induce gene expression of key factors in both ubiquitin proteasome system (UPS) $(32,69)$ and the autophagy lysosome pathways (45).

\section{Concluding remarks}

Exercise training promotes a range of beneficial adaptations in the skeletal muscle including increased capillarization, fibre type plasticity, hypertrophy and function. All these adaptations are a result of exercise stimuli that challenges muscle homeostasis by activating networks of signalling molecules. Activation of kinases and pre-transcriptional regulation occurs rapidly during exercise and recovery, whereas protein transcription is subsequently regulated. Intensity, duration, and mode of the exercise stimuli collectively contribute to the relative activation and the magnitude of activated pathways and downstream targets $(9,12,13)$. All these parameters have to be considered when designing exercise studies, so results are comparable and can advance knowledge in the area. Future studies on the molecular mechanisms of exercise induced satellite cell myogenic capacity in COPD patients are fundamental for designing pharmacological and exercise training interventions aiming to address limitations to exerciseinduced muscle adaptations.

\section{Acknowledgements}

DC Simoes is supported by the University of Northumbria Multidisciplinary Research Theme (MDRT).

\section{Footnote}

Conflicts of Interest: The authors have no conflicts of interest to declare.

\section{References}

1. Vogelmeier CF, Criner GJ, Martinez FJ, et al. Global Strategy for the Diagnosis, Management, and Prevention of Chronic Obstructive Lung Disease 2017 Report: GOLD Executive Summary. Arch Bronconeumol 2017;53:128-49.
2. Barnes PJ, Celli BR. Systemic manifestations and comorbidities of COPD. Eur Respir J 2009;33:1165-85.

3. Barreiro E, Peinado VI, Galdiz JB, et al. Cigarette smokeinduced oxidative stress: A role in chronic obstructive pulmonary disease skeletal muscle dysfunction. Am J Respir Crit Care Med 2010;182:477-88.

4. Kim HC, Mofarrahi M, Hussain SN. Skeletal muscle dysfunction in patients with chronic obstructive pulmonary disease. Int J Chron Obstruct Pulmon Dis 2008;3:637-58.

5. Gea J, Agusti A, Roca J. Pathophysiology of muscle dysfunction in COPD. J Appl Physiol (1985) 2013;114:1222-34.

6. Hopkinson NS, Tennant RC, Dayer MJ, et al. A prospective study of decline in fat free mass and skeletal muscle strength in chronic obstructive pulmonary disease. Respir Res 2007;8:25.

7. Patel MS, Natanek SA, Stratakos G, et al. Vastus lateralis fiber shift is an independent predictor of mortality in chronic obstructive pulmonary disease. Am J Respir Crit Care Med 2014;190:350-2.

8. Gouspillou G, Bourdel-Marchasson I, Rouland R, et al. Mitochondrial energetics is impaired in vivo in aged skeletal muscle. Aging Cell 2014;13:39-48.

9. Maltais F, Decramer M, Casaburi R, et al. An official American Thoracic Society/European Respiratory Society statement: update on limb muscle dysfunction in chronic obstructive pulmonary disease. Am J Respir Crit Care Med 2014;189:e15-62.

10. Vogiatzis I, Simoes DCM, Stratakos G, et al. Effect of pulmonary rehabilitation on muscle remodelling in cachectic patients with COPD. Eur Respir J 2010;36:301-10.

11. Vogiatzis I, Terzis G, Stratakos G, et al. Effect of pulmonary rehabilitation on peripheral muscle fiber remodeling in patients with COPD in GOLD stages II to IV. Chest 2011;140:744-52.

12. Coffey VG, Hawley JA. The molecular bases of training adaptation. Sports Med 2007;37:737-63.

13. Fluck M, Hoppeler H. Molecular basis of skeletal muscle plasticity--from gene to form and function. Rev Physiol Biochem Pharmacol 2003;146:159-216.

14. Cartee GD, Hepple RT, Bamman MM, et al. Exercise Promotes Healthy Aging of Skeletal Muscle. Cell Metab 2016;23:1034-47.

15. Egan B, Zierath JR. Exercise metabolism and the molecular regulation of skeletal muscle adaptation. Cell Metab 2013;17:162-84.

16. Costes F, Gosker H, Feasson L, et al. Impaired exercise 
training-induced muscle fiber hypertrophy and Akt/ mTOR pathway activation in hypoxemic patients with COPD. J Appl Physiol (1985) 2015;118:1040-9.

17. Vogiatzis I, Stratakos G, Simoes DC, et al. Effects of rehabilitative exercise on peripheral muscle TNF alpha, IL-6, IGF-I and MyoD expression in patients with COPD. Thorax 2007;62:950-6.

18. Guzun R, Aguilaniu B, Wuyam B, et al. Effects of training at mild exercise intensities on quadriceps muscle energy metabolism in patients with chronic obstructive pulmonary disease. Acta Physiol (Oxf) 2012;205:236-46.

19. De Brandt J, Spruit MA, Derave W, et al. Changes in structural and metabolic muscle characteristics following exercise-based interventions in patients with COPD: a systematic review. Expert Rev Respir Med 2016;10:521-45.

20. Lanza IR, Short DK, Short KR, et al. Endurance exercise as a countermeasure for aging. Diabetes 2008;57:2933-42.

21. Robinson MM, Dasari S, Konopka AR, et al. Enhanced Protein Translation Underlies Improved Metabolic and Physical Adaptations to Different Exercise Training Modes in Young and Old Humans. Cell Metab 2017;25:581-92.

22. Vogiatzis I, Terzis G, Nanas S, et al. Skeletal muscle adaptations to interval training in patients with advanced COPD. Chest 2005;128:3838-45.

23. Saltin B, Henriksson J, Nygaard E, et al. Fiber types and metabolic potentials of skeletal muscles in sedentary man and endurance runners. Ann N Y Acad Sci 1977;301:3-29.

24. Natanek SA, Gosker HR, Slot IG, et al. Heterogeneity of quadriceps muscle phenotype in chronic obstructive pulmonary disease (Copd); implications for stratified medicine? Muscle Nerve 2013;48:488-97.

25. Gouzi F, Prefaut C, Abdellaoui A, et al. Blunted muscle angiogenic training-response in COPD patients versus sedentary controls. Eur Respir J 2013;41:806-14.

26. Man WD, Soliman MG, Nikoletou D, et al. Nonvolitional assessment of skeletal muscle strength in patients with chronic obstructive pulmonary disease. Thorax 2003;58:665-9.

27. Gea JG, Pasto M, Carmona MA, et al. Metabolic characteristics of the deltoid muscle in patients with chronic obstructive pulmonary disease. Eur Respir J 2001;17:939-45.

28. Levine S, Gregory C, Nguyen T, et al. Bioenergetic adaptation of individual human diaphragmatic myofibers to severe COPD. J Appl Physiol (1985) 2002;92:1205-13.

29. Levine S, Kaiser L, Leferovich J, et al. Cellular adaptations in the diaphragm in chronic obstructive pulmonary disease. N Engl J Med 1997;337:1799-806.
30. Bogdanis GC. Effects of physical activity and inactivity on muscle fatigue. Front Physiol 2012;3:142.

31. Bergeron R, Ren JM, Cadman KS, et al. Chronic activation of AMP kinase results in NRF-1 activation and mitochondrial biogenesis. Am J Physiol Endocrinol Metab 2001;281:E1340-6.

32. Sandri M, Sandri C, Gilbert A, et al. Foxo transcription factors induce the atrophy-related ubiquitin ligase atrogin-1 and cause skeletal muscle atrophy. Cell 2004;117:399-412.

33. Long YC, Widegren U, Zierath JR. Exercise-induced mitogen-activated protein kinase signalling in skeletal muscle. Proc Nutr Soc 2004;63:227-32.

34. Choi I, Lee K, Kim M, et al. Differential activation of stress-responsive signalling proteins associated with altered loading in a rat skeletal muscle. J Cell Biochem 2005;96:1231-43.

35. Yu M, Stepto NK, Chibalin AV, et al. Metabolic and mitogenic signal transduction in human skeletal muscle after intense cycling exercise. J Physiol 2003;546:327-35.

36. Bernet JD, Doles JD, Hall JK, et al. p38 MAPK signaling underlies a cell-autonomous loss of stem cell self-renewal in skeletal muscle of aged mice. Nat Med 2014;20:265-71.

37. Childs SG. Muscle wasting. Orthop Nurs 2003;22:251-7; quiz 8-9.

38. Pitta F, Troosters T, Spruit MA, et al. Characteristics of physical activities in daily life in chronic obstructive pulmonary disease. Am J Respir Crit Care Med 2005;171:972-7.

39. Lemire BB, Debigare R, Dube A, et al. MAPK signaling in the quadriceps of patients with chronic obstructive pulmonary disease. J Appl Physiol (1985) 2012;113:159-66.

40. Remels AH, Schrauwen P, Broekhuizen R, et al. Peroxisome proliferator-activated receptor expression is reduced in skeletal muscle in COPD. Eur Respir J 2007;30:245-52.

41. Puente-Maestu L, Lazaro A, Tejedor A, et al. Effects of exercise on mitochondrial DNA content in skeletal muscle of patients with COPD. Thorax 2011;66:121-7.

42. Terzis G, Georgiadis G, Stratakos G, et al. Resistance exercise-induced increase in muscle mass correlates with p70S6 kinase phosphorylation in human subjects. Eur J Appl Physiol 2008;102:145-52.

43. Bodine SC, Latres E, Baumhueter S, et al. Identification of ubiquitin ligases required for skeletal muscle atrophy. Science 2001;294:1704-8.

44. Stitt TN, Drujan D, Clarke BA, et al. The IGF-1/ PI3K/Akt pathway prevents expression of muscle 
atrophy-induced ubiquitin ligases by inhibiting FOXO transcription factors. Mol Cell 2004;14:395-403.

45. Mammucari C, Milan G, Romanello V, et al. FoxO3 controls autophagy in skeletal muscle in vivo. Cell Metab 2007;6:458-71.

46. Zhao J, Brault JJ, Schild A, et al. FoxO3 coordinately activates protein degradation by the autophagic/lysosomal and proteasomal pathways in atrophying muscle cells. Cell Metab 2007;6:472-83.

47. Favier FB, Costes F, Defour A, et al. Downregulation of $\mathrm{Akt} / \mathrm{mammalian}$ target of rapamycin pathway in skeletal muscle is associated with increased REDD1 expression in response to chronic hypoxia. Am J Physiol Regul Integr Comp Physiol 2010;298:R1659-66.

48. Radom-Aizik S, Kaminski N, Hayek S, et al. Effects of exercise training on quadriceps muscle gene expression in chronic obstructive pulmonary disease. J Appl Physiol (1985) 2007;102:1976-84.

49. Sandri M. Signaling in muscle atrophy and hypertrophy. Physiology (Bethesda) 2008;23:160-70.

50. Philp A, Hamilton DL, Baar K. Signals mediating skeletal muscle remodeling by resistance exercise: PI3-kinase independent activation of mTORC1. J Appl Physiol (1985) 2011;110:561-8.

51. Adams GR, McCue SA. Localized infusion of IGF-I results in skeletal muscle hypertrophy in rats. J Appl Physiol (1985) 1998;84:1716-22.

52. Rennie MJ, Wackerhage H, Spangenburg EE, et al. Control of the size of the human muscle mass. Annu Rev Physiol 2004;66:799-828.

53. Adams GR, Bamman MM. Characterization and regulation of mechanical loading-induced compensatory muscle hypertrophy. Compr Physiol 2012;2:2829-70.

54. Allen DL, Roy RR, Edgerton VR. Myonuclear domains in muscle adaptation and disease. Muscle Nerve 1999;22:1350-60.

55. Theriault ME, Pare ME, Maltais F, et al. Satellite cells senescence in limb muscle of severe patients with COPD. PLoS One 2012;7:e39124.

56. Constantin D, Menon MK, Houchen-Wolloff L, et al. Skeletal muscle molecular responses to resistance training and dietary supplementation in COPD. Thorax 2013;68:625-33

57. Lewis MI, Fournier M, Storer TW, et al. Skeletal muscle adaptations to testosterone and resistance training in men with COPD. J Appl Physiol (1985) 2007;103:1299-310.

58. Annunziata M, Granata R, Ghigo E. The IGF system. Acta Diabetol 2011;48:1-9.
59. Zanou N, Gailly P. Skeletal muscle hypertrophy and regeneration: interplay between the myogenic regulatory factors (MRFs) and insulin-like growth factors (IGFs) pathways. Cell Mol Life Sci 2013;70:4117-30.

60. Hussain SN, Sandri M. Role of autophagy in COPD skeletal muscle dysfunction. J Appl Physiol (1985) 2013;114:1273-81.

61. Jagoe RT, Engelen MP. Muscle wasting and changes in muscle protein metabolism in chronic obstructive pulmonary disease. Eur Respir J Suppl 2003;46:52s-63s.

62. Puig-Vilanova E, Rodriguez DA, Lloreta J, et al. Oxidative stress, redox signaling pathways, and autophagy in cachectic muscles of male patients with advanced COPD and lung cancer. Free Radic Biol Med 2015;79:91-108.

63. Fermoselle C, Rabinovich R, Ausin P, et al. Does oxidative stress modulate limb muscle atrophy in severe COPD patients? Eur Respir J 2012;40:851-62.

64. Debigare R, Cote CH, Maltais F. Ubiquitination and proteolysis in limb and respiratory muscles of patients with chronic obstructive pulmonary disease. Proc Am Thorac Soc 2010;7:84-90.

65. Debigare R, Maltais F, Cote $\mathrm{CH}$, et al. Profiling of mRNA expression in quadriceps of patients with COPD and muscle wasting. COPD 2008;5:75-84.

66. Doucet M, Russell AP, Leger B, et al. Muscle atrophy and hypertrophy signaling in patients with chronic obstructive pulmonary disease. Am J Respir Crit Care Med 2007;176:261-9.

67. Guo Y, Gosker HR, Schols AM, et al. Autophagy in locomotor muscles of patients with chronic obstructive pulmonary disease. Am J Respir Crit Care Med 2013;188:1313-20.

68. Doucet M, Dube A, Joanisse DR, et al. Atrophy and hypertrophy signalling of the quadriceps and diaphragm in COPD. Thorax 2010;65:963-70.

69. Gielen S, Sandri M, Kozarez I, et al. Exercise training attenuates MuRF-1 expression in the skeletal muscle of patients with chronic heart failure independent of age: the randomized Leipzig Exercise Intervention in Chronic Heart Failure and Aging catabolism study. Circulation 2012;125:2716-27.

Cite this article as: Simoes DC, Vogiatzis I. Can muscle protein metabolism be specifically targeted by exercise training in COPD? J Thorac Dis 2018;10(Suppl 12):S1367-S1376. doi: 10.21037/jtd.2018.02.67 